\title{
On the Error Performance of Coding and Equalization in Low-Complexity Ultra-Wideband Communication Systems
}

\author{
Robert H. Morelos-Zaragoza
}

\begin{abstract}
In this paper, the performance of various channel coding schemes is investigated in pulse-based ultra-wideband (UWB) communication systems for applications in short-range indoor environments. Pulse-based binary (BPSK) modulation and decision-feedback equalization (DFE) is considered. Concatenated adaptive equalization and coding is explored as an alternative to the more complex and often impractical joint coding and equalization. A block length of approximately 1000 bits is considered in this paper as it results in a static channel with minimal latency while still yielding relatively good error performance. The error performance of a previously proposed turbo product code (TPC), based on two identical Hamming $(31,26)$ codes, is simulated and compared with that of other channel coding schemes of similar rate and code length. These include a regular LDPC $(1057,813)$ code, a memory-6 rate-3/4 punctured convolutional code, a Reed-Solomon $(127,89)$ code and a concatenated (off-the-shelf) code with a Reed-Solomon $(255,239)$ outer code and a memory-6 rate-3/4 punctured convolutional inner code. The inclusion of the concatenated ReedSolomon scheme serves as a reference, as this is an off-the-shelf classical and still popular solution. The simulation results show that, among the coding schemes considered, the LDPC code offers the best error performance.
\end{abstract}

Index Terms-Wireless communications, equalization, error correcting codes.

\section{INTRODUCTION}

In recent years, a significant research effort has been devoted to the study of ultra-wideband (UWB) communication systems [1]-[2]. The basic principle behind ultra-wideband communications was first used in radar systems over thirty years ago [3]. The UWB concept was used to develop impulse radio, where baseband pulses are transmitted over the channel [4]. This technique greatly simplifies the transmitter and receiver designs; however, the transmitted bandwidth extends to the gigaHertz range. Any application of UWB technology must conform to the regulations imposed on radio-frequency transmissions. In the United States, these regulations are established and enforced by the Federal Communications Commission (FCC). The FCC regulation [5] permits transmission of signals with $-10 \mathrm{~dB}$ bandwidths that lie in the 3.1 to $10.6 \mathrm{GHz}$ band, provided that the transmitted signals have an effective isotropic radiated power (EIRP) below $-41.3 \mathrm{dBm}$ in this band and a minimum bandwidth of $500 \mathrm{MHz}$. The proposed

Manuscript received May 12, 2006; revised August 25, 2006.

Robert H. Morelos - Zaragoza is with the Department of Electrical Engineering, San José State University, San Jose, CA 95192-0084 U.S.A. (e-mail: R.Morelos-Zaragoza@IEEE.org).
IEEE 802.15.3a standard defines the requirements of a wireless personal-area network (WPAN) communication system [6]. These requirements include a bit rate of at least $110 \mathrm{Mbps}$ at a distance of up to $10 \mathrm{~m}$ and $200 \mathrm{Mbps}$ at up to $4 \mathrm{~m}$, with desired rates up to $480 \mathrm{Mbps}$. A pulse-based UWB approach is a good candidate for meeting the WPAN requirements.

In this paper, the error performance of error correcting coding is investigated in a UWB communication system with adaptive decision-feedback equalization and pulse-based modulation for applications in short-range indoor environments. Performance is measured using the reference UWB multipath channel models generated by the IEEE 802.15.3a standard group [7]. A communication system is considered here in which known symbols are used to train the adaptive equalizer. To reduce the variation in the bit error rate with respect to changing multipath channel conditions, a turbo product code (TPC) with two component $(31,26,3)$ Hamming codes was proposed in [9]. The purpose of the work reported here is to consider other channel coding schemes of similar rate, such as a low-complexity LDPC code, a Reed-Solomon code and a concatenated code, and to evaluate and compare their error performance with respect to the TPC code previously proposed.

The rest of the paper is organized as follows: In section II, the UWB communication system model used in this study is presented. It is shown that an equivalent symbol-spaced UWB channel model is obtained between the input to the pulse shaping filter and the output of the matched filter. Section III considers the performance of suboptimal adaptive equalizers as low-complexity alternatives to the Viterbi equalizer. Performance is studied in terms of equalizer length and sensitivity to different channel realizations. Simulation results, presented in section IV-A, of combinations of adaptive equalizers and a turbo product code, illustrate the benefits of using channel coding as an effective way to improve performance and reduce sensitivity to channel variations.

\section{UWB COMMUNICATION SYSTEM MODEL}

Fig. 1 shows a block diagram of the binary pulse-based UWB system under consideration. The rate of the BPSK modulator is $250 \mathrm{Mbps}$, i.e., a symbol period $T=4 \mathrm{~ns}$. Channel encoding with coding rate equal to 0.7 results in an effective information rate equal to $175.86 \mathrm{Mbps}$. The output of the modulator is a binary-valued sequence $\left\{s_{n}\right\}$, with 


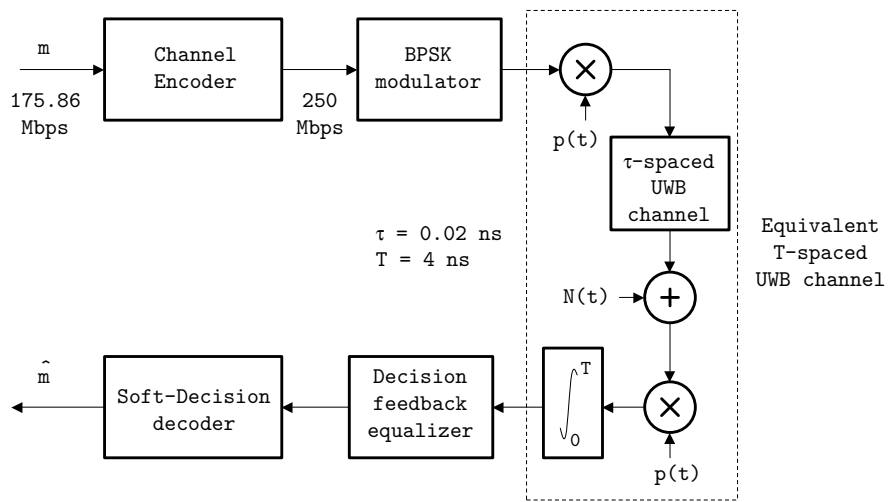

Fig. 1. A pulse-based UWB communication system with channel coding and decision-feedback equalization.

$s_{n} \in\{-1,+1\}$. A wideband unit-energy real-valued pulse shape $p(t)$ is employed such that the output of the transmit filter is given by

$$
s(t)=\sum_{n=-\infty}^{\infty} s_{n} p(t-n T) .
$$

It is important to note that the pulse shape $p(t)$ can be either a single UWB pulse or a sequence of UWB pulses (as in spreadspectrum systems) with good autocorrelation properties.

The UWB channel model employed in this work is fully compliant with that of the IEEE 802.15.3a group [7]. The model is based on a modified Saleh-Valenzuela model [8] with a lognormal distribution rather than a Rayleigh distribution for the multipath gain magnitudes. UWB channel models have been classified into four different modes: CM1 through CM4, with CM1 being a mild condition corresponding to short distances and line-of-sight and CM4 associated with extreme multipath conditions. To produce the results reported in this paper, a channel sampling time $\tau=0.02 \mathrm{~ns}$ was used. The UWB channel impulse response (CIR) is given by

$$
h(t)=\sum_{\ell=0}^{L-1} \alpha_{\ell} \delta\left(t-\tau_{\ell}\right),
$$

where $\alpha_{\ell}$ and $\tau_{\ell}$ are the gain and delay of the $\ell$-th channel path, for $\ell=0,1, \cdots, L-1$. The path delays can be expressed as multiples of the sampling time: $\tau_{\ell}=m_{\ell} \tau$, where for $0 \leq \ell \leq$ $L-1, m_{\ell}$ is a positive integer and $m_{0}<m_{1}<\cdots<m_{L-1}$. The delay spread of the channel is equal to $\tau_{L-1}$.

It is assumed here that the noise process introduced at the receiver and denoted by $N(t)$ is a zero-mean AWGN process with double-sided power spectral density $N_{0} / 2 \mathrm{~W} / \mathrm{Hz}$. Under this assumption, the output of the matched filter at $t=m T$ can be expressed as

$$
\begin{aligned}
Y_{m} & =\sum_{\ell=0}^{L-1} \alpha_{\ell} \int_{(m-1) T}^{m T} s\left(t-\tau_{\ell}\right) p(t) d t+W \\
& =\sum_{n=-\infty}^{\infty} s_{n} \sum_{\ell=0}^{L-1} \alpha_{\ell} \int_{(m-1) T}^{m T} p\left(t-\tau_{\ell}-n T\right) p(t) d t
\end{aligned}
$$

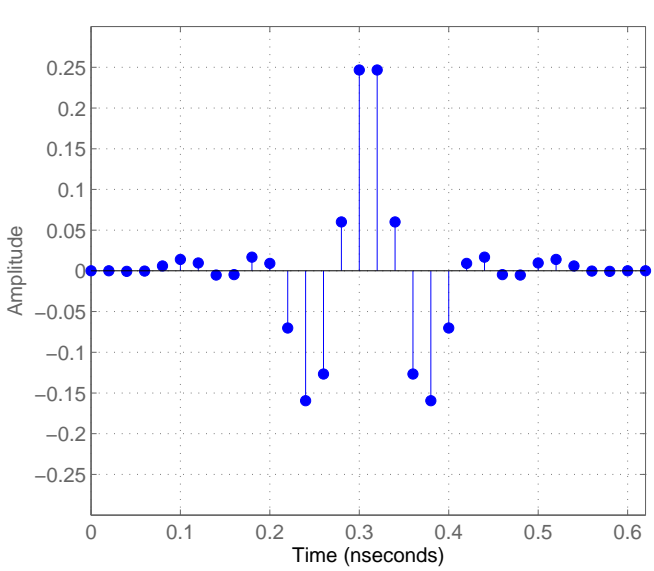

Fig. 2. UWB pulse shape.

$$
=\sum_{i=0}^{P-1} \beta_{i} s_{m M-i}+W,
$$

where $P=\lfloor T / \tau\rfloor=200$ is the ratio of the channel sampling rate to the symbol rate, or the number of sampling periods within a symbol period, and $W$ is a zero-mean Gaussian random variable of variance equal to $\sigma^{2}=N_{0} / 2$. This results in an equivalent symbol-spaced UWB CIR which is given by

$$
g(t)=\sum_{j=0}^{J-1} \beta_{j} \delta(t-j T),
$$

where $J=\left\lfloor\tau_{L-1} / T\right\rfloor$ is the ratio of the channel delay spread to the symbol period. The path gains of the $T$-spaced CIR, $\beta_{j}$, depend on the autocorrelation function of $p(t)$ and on the path gains of the $\tau$-spaced CIR $\alpha_{\ell}$, over the period $[(m-J+1+$ $j) T,(m-J+1+j+1) T]$, for $0 \leq j \leq J-1$. Figure 2 shows the shape of the pulse used in the simulations. In figure 3 , the CIR of a sample realization of UWB channel type CM4 is illustrated. It is assumed that the channel remains static for a period of time in the order of thousands of symbols. This is a realistic assumption in an indoor environment with walking speeds.

The outputs of the matched filter constitute the inputs to an adaptive decision-feedback equalizer. The equalized output symbols are then delivered to a soft-decision decoder for the channel coding scheme under consideration. The decoder may or may not be iterative, depending on the class of channel code being considered. Finally, the outputs of the channel decoder constitute the estimated information bits $\hat{m}$.

\section{ADAPTIVE EQUALIZATION}

In this section, the performance of adaptive equalizers for the binary pulse-based UWB communication system outlined in the previous section is considered. As made clearly evident by Fig. 3, data transmission over the equivalent symbol-spaced UWB channel results in a severe amount of intersymbol interference (ISI). Although the optimum receiver is known to be a Viterbi equalizer (VE) [10], its complexity grows exponentially with the length $J$ of the symbol-spaced ISI 

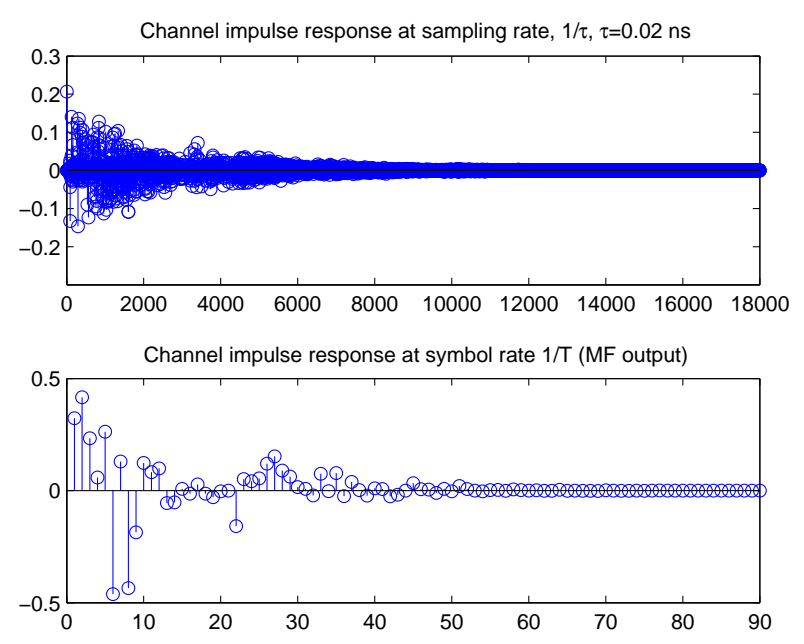

Fig. 3. A UWB CM4-type CIR realization with $P=200$.

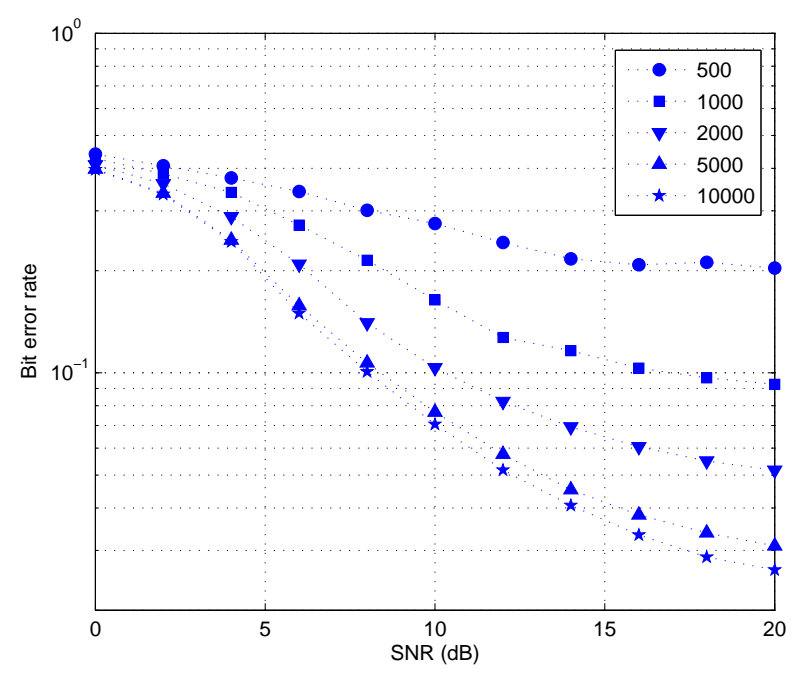

Fig. 4. Performance of a 95-tap linear equalizer with different training sequence lengths. CM4 channel.

channel. Moreover, even with techniques such as a reducedstate trellises [11], complexity remains an exponential function of $J$. An alternative approach to reduce the complexity of the VE solution is the use of adaptive equalization techniques [12][14]. At the cost of a performance loss, the complexity of adaptive equalizers is a linear function of $J$. Consequently, it becomes of practical interest to study the performance of adaptive equalizers in UWB communications.

An adaptive equalizer needs to be trained, either by using a pilot sequence and estimating the channel to provide an initial setting of the coefficients [15], or by the use of a training sequence. For UWB applications, fast acquisition becomes important and channel estimation is the preferred method. In the simulation results presented below, we used a training sequence of 10000 symbols to initialize the equalizer. To provide a justification for this choice of training sequence length, Figs. 4 and 5 show the performance of 95-tap linear and nonlinear adaptive equalizers for an extreme multipath

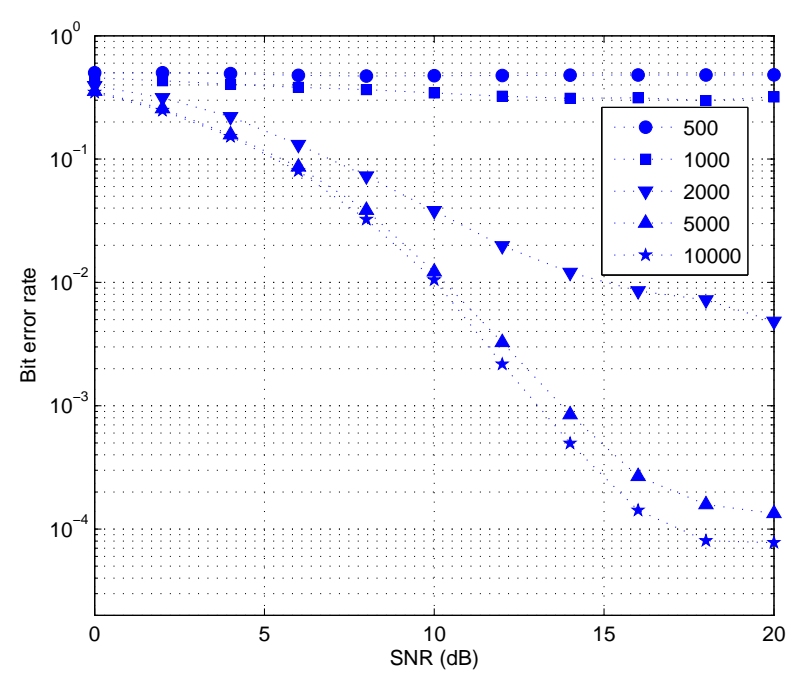

Fig. 5. Performance of a 95-tap nonlinear equalizer $\left(M=63, M_{f}=32\right)$ with different training sequence lengths. CM4 channel.

channel (CM4 type), respectively. Although the simulation results in this work are obtained using a training sequence, the results and conclusions can be extended to the case of channel estimation using pilot sequences [15].

\section{A. Linear equalization}

An adaptive linear equalizer (LE) is an FIR filter in which $M$ tap coefficients $\left\{w_{m}\right\}$ are updated in order to optimize a given cost function. Here, we use the mean square error (MSE) as the cost function. The coefficients are modified in order to minimize the MSE using the least mean square (LMS) algorithm, as follows. The output of the LE at time $t=n T$ is

$$
c_{n}=\sum_{m=0}^{M-1} w_{m} Y_{m}
$$

Let the error sample be defined as $e_{n}=\hat{c}_{n}-c_{n}$, where $\hat{c}_{n}=$ $\operatorname{sgn}\left(c_{n}\right)$ is the output of the BPSK slicer in decision-directed mode and a known symbol in training (data-aided) mode. Then the coefficients are updated via

$$
w_{m}(n+1)=\mu w_{m}(n)+\Delta Y_{n} e_{n}
$$

where $\Delta$ is the step size and $\mu$ is the forgetting factor, $0<$ $\mu \leq 1$. Simulation results, not reported here, show that $\Delta=$ 0.00085 and $\mu=0.75$ are good choices.

Fig. 6 shows the performance of an LE with different numbers of taps $M$ over a UWB channel type CM1. From these results, it evident that the performance variation is relatively small, provided that the equalizer length $M$ is larger than the ISI length $J$ of the UWB channel. However, note that over an extreme multipath density channel (type CM4), Fig. 7 shows that performance may degrade considerably, not only in comparison to the CM1 channel, but also increasing as a function of $M$. 


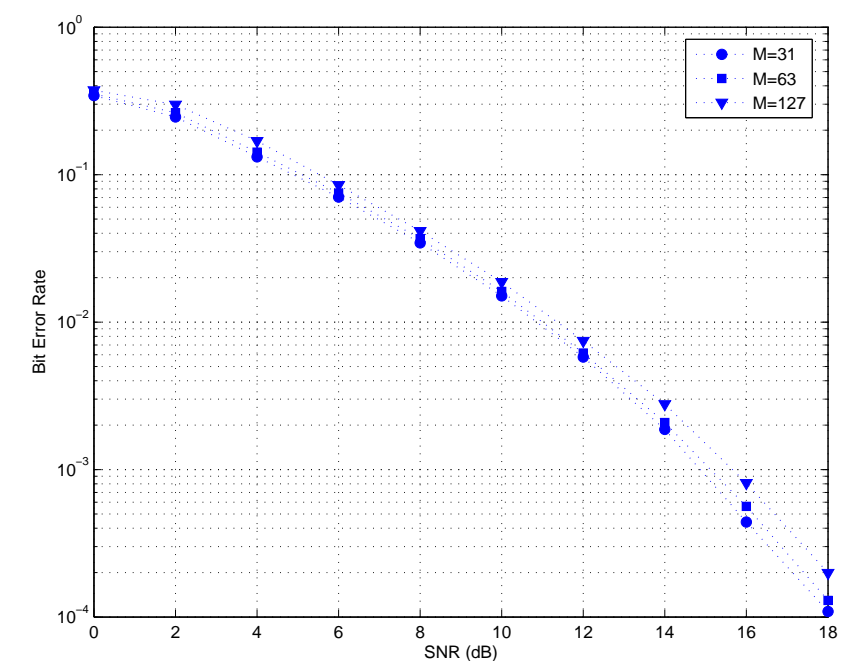

Fig. 6. Linear equalizer with different number of taps. CM1 channel.

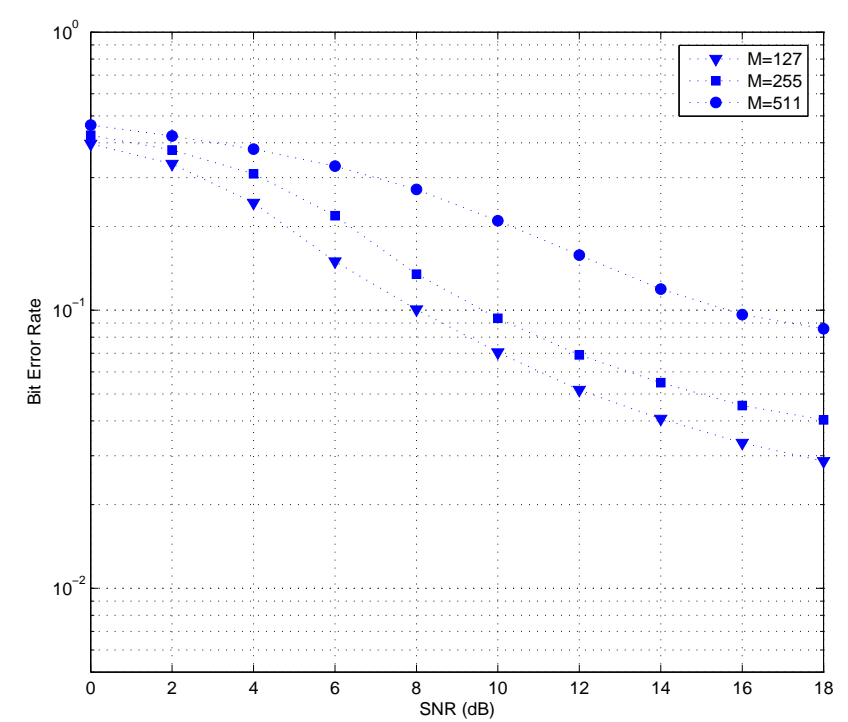

Fig. 7. Linear equalizer with different number of taps. CM4 channel.

\section{B. Decision-feedback equalization}

Decision-feedback equalizers for wideband communications have been studied extensively [15]-[21]. A brief overview of the fundamental idea is presented next. Let the error be defined as $e_{n}=\hat{c}_{n}-c_{n}$, where $\hat{c}_{n}$ is either the estimated symbol at the output of a BPSK slicer, $\hat{c}_{n}=\operatorname{sgn}\left(c_{n}\right)$, or a known symbol in training (data-aided) mode. The output of the equalizer at time $t=n T$ is given by

$$
c_{n}=\sum_{m=0}^{M-1} w_{m} Y_{n-m}+\sum_{\ell=1}^{M_{f}} v_{m} \hat{c}_{n-\ell},
$$

and is based on the $M$ most recent matched filter outputs $\left\{Y_{n-m}, m=0,1, \cdots, M-1\right\}$ and on the $M_{f}$ most recent decisions $\left\{\hat{c}_{n-\ell}, \ell=1,2, \cdots, M_{f}\right\}$ (or known symbols in

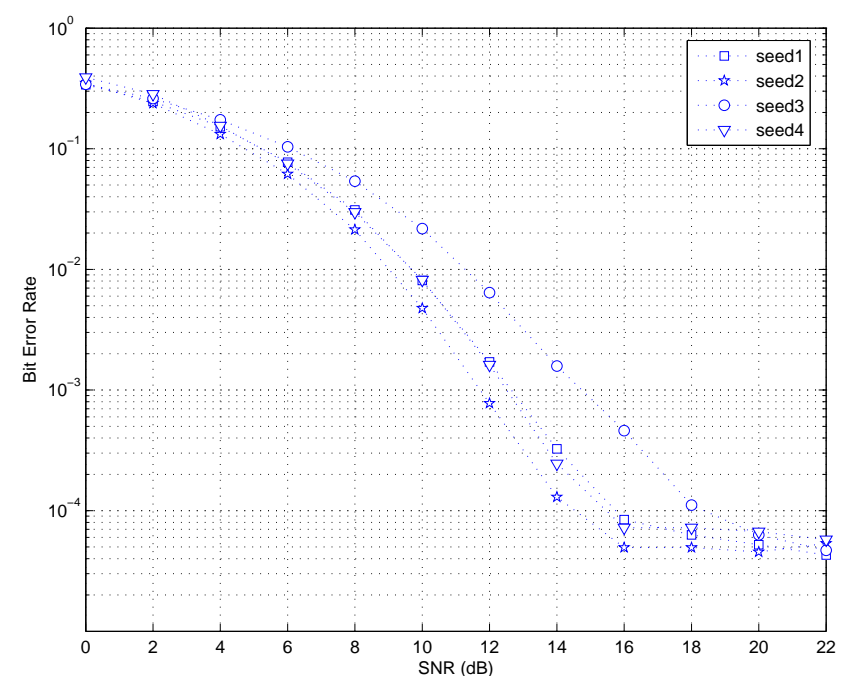

Fig. 8. DFE with four realizations of a CM4 channel.

training mode). The feedforward and feedback coefficients are updated via the LMS algorithm as follows:

$$
\begin{aligned}
w_{m}(n+1) & =\mu w_{m}(n)+\Delta Y_{n} e_{n} \\
v_{m}(n+1) & =\mu v_{m}(n)+\Delta Y_{n} e_{n} .
\end{aligned}
$$

In the case of the pulse-based UWB communication system under consideration, the setting of the adaptive DFE parameters was studied based on computer simulations in [9], were it was found that the values $M=63, M_{f}=32, \Delta=0.00085$ and $\mu=0.75$ gave good performance across all UWB channel types CM1 through CM4.

A strong motivation for the need for channel coding, in the context of pulse-based UWB systems with adaptive equalization, can be obtained from the sensitivity of the performance of an adaptive DFE with respect to four different channel realizations of a CM4-type UWB channel, as illustrated in Fig. 8. The channel type CM4 models extreme multipath conditions in an indoor environment [7]. Two important observations can be made based on this result: (1) An irreducible error floor may appear due to the fact that DFE cannot completely remove the ISI; and (2) the signal-to-noise ratio (SNR) required to achieve a particular target bit error rate (BER) is expected to have a range of values of at least $3 \mathrm{~dB}$. Similar studies [9] for the other UWB channel types, ranging from mild multipath conditions (CM1 type) to dense multipath conditions (CM3 type), show that the SNR variation grows with the multipath density or maximum delay spread, i.e., least variation for CM1 channels and most variation for CM4 channels.

A practical way to reduce the SNR variation in error performance of pulse-based UWB communication systems with adaptive equalization is by the use of powerful error correcting codes. This is the topic of the next section.

\section{Channel CODING SCHEMES}

In this section, a comparison of the error performances of various error correcting codes applied to a pulse-based BPSK 
modulated UWB system with DFE is presented. In order to produce a meaningful comparison with the turbo product coding (TPC) scheme proposed in [9], the coding rate is set to $R_{c}=0.7$. In particular, the following error correcting codes are investigated:

1) A TPC $(31,26)^{2}$ code using two component binary Hamming $(31,26,3)$ codes

2) A regular low-density parity-check (LDPC) $(1057,813)$ code with node degrees $J=3$ and $K=13$

3) A concatenated code with an outer Reed-Solomon $(255,239)$ code over $G F\left(2^{8}\right)$ and an inner memory6 rate- $3 / 4$ binary punctured convolutional code (PCC) based on a rate- $1 / 2$ convolutional encoder with generators, in octal notation, $(171,133)$

4) A binary-image $(889,623)$ code obtained from a ReedSolomon $(127,89)$ code over $G F\left(2^{7}\right)$

The selection of these particular codes is based on practical considerations, which include availability and small latency. It is emphasized that no attempt was made to achieving performance close to capacity, but rather to obtaining a good performance with practical codes of relatively short lengths and similar rate. Regular LDPC codes are known to perform better than irregular LDPC codes at high SNR values (error floor region). However, irregular codes provide better performance at low SNR values (waterfall region). Here, a regular code is selected here mainly because it may be easier to encode and decode as its parity-check matrix and underlying Tanner graph have regular structure. The concatenated Reed-Solomon scheme serves as a reference. It has the same rate as the TPC scheme and constitutes a classical solution.

\section{A. Turbo product coding with binary Hamming $(31,26)$ codes}

To improve upon the error performance of adaptive equalizers, a turbo product code (TPC) [23] was proposed and its error performance studied in [9]. This TPC is constructed from two component $(31,26,3)$ Hamming codes and has a coding rate $R=(26 / 31)^{2}=0.7034$. Henceforth, we refer to this code as TPC $(31,26)^{2}$. Iterative soft-input soft-output (SISO) decoding with the Chase type-II algorithm [24] and four decoding iterations was employed. This channel coding approach is attractive from a practical perspective because of its very low complexity compared to other types of codes and decoding algorithms, while at the same time exhibiting turbolike error performance. It is interesting to note that a similar TPC scheme, using a shortened extended Hamming $(31,25,4)$ code, has been adopted in the IEEE 802.16 2004 standard for fixed broadband wireless communications [25].

1) Iterative decoding with Chase type-II algorithm: In the Chase type-II decoding algorithm, the equalizer outputs $c_{n}$ are scored by their reliability values $\left|c_{n}\right|$. A bit position $n$ is said to be reliable if the value of $\left|c_{n}\right|$ is high. Error patterns $\bar{e}$ are constructed for those code positions with low reliability values. For each error pattern $\bar{e}$, a noisy test vector $\bar{r}=\bar{z}+\bar{e}$ is generated, where $z_{n}=\operatorname{sgn}\left(c_{n}\right)$ is the $n$-th component of the hard-decision received vector. The closest codeword $\bar{v}$ to the test vector $\bar{r}$ is determined via a harddecision decoder. For a $(31,26,3)$ Hamming code, harddecision decoding is extremely simple, using a combinatorial circuit to implement a syndrome look-up table. Moreover, since the minimum Hamming distance is equal to 3, Chase type-II algorithm reduces to Wagner decoding [26], whereby only one test vector is generated by simply complementing the bit in the least reliable position. This reduces decoding complexity dramatically, compared to that required for decoding extended Hamming codes. At each decoding iteration, soft-outputs are generated using the two closest codewords, $\bar{v}_{1}$ and $\bar{v}_{2}$, to $\bar{z}$. In the event that these codewords are identical, the procedure suggested in [27] is used. In the simulations reported below, four iterations of decoding are performed as suggested by Pyndhia [23]. Increasing weights are used to modify the reliability correction factors when feeding back the extrinsic information in the iterative SISO decoder. This helps to speed up convergence of the decoding algorithm.

\section{B. Regular LDPC $(1057,813)$ code}

This LDPC code was constructed by computer search [29]. In the context of LDPC coding, this is considered a very short code. Although in the low SNR region irregular LDPC codes are known to perform better, they require optimization of the node degree distribution. A regular code is selected here as it has the advantage that it is easier to encode and decode because of the regular structure of its parity-check matrix and underlying Tanner graph. The problem of designing good LDPC codes for applications in UWB systems (either in the waterfall region or in the error floor region) remains as a topic of future research interest.

A Tanner graph $\mathcal{T}$ a bipartite graph with two types of nodes: Variable nodes and check nodes [28]. $\mathcal{T}$ is obtained by regarding $H$ as its incidence matrix. Each row of $H$ specifies how variable nodes are connected to a check node. Conversely, each column of $H$ specifies how check nodes are connected to a variable node. For the regular LDPC $(1057,813)$ code, the Hamming weight of each column of $H$ is $J=3$ and each row of $H$ has Hamming weight $K=13$. The variable node degrees and check node degrees of the associated regular Tanner graph $\mathcal{T}$ are $J=3$ and $K=13$. Iterative belief-propagation (IBP) decoding can be applied to this graph [30].

1) IBP decoding: In IBP decoding, the received symbols from the channel (also known as channel log-likelihood ratio values) are used as initial metrics of the variable nodes. It is important to point out that in computing this initial metrics, the value of the variance $\sigma_{N}^{2}=N_{0} / 2$ of the underlying AWGN process samples needs to be known. The variable node metrics are then sent to check nodes in a top-down messagepassing step and new metrics computed for the check nodes. An iteration is completed with a bottom-up message-passing step in which the check node metrics are sent back to the variable nodes. Details on IBP decoding and variations thereof can be found in [22]. In the simulation results reported in a later section, the maximum number of iterations is set to four. This is done for practical considerations of decoding latency. An increase in the number of iterations results in better 
TABLE I

SUMMARY OF CODING SCHEMES

\begin{tabular}{|c|ccc|}
\hline Scheme & Length & Dimension & Rate \\
\hline A & 961 & 676 & 0.703 \\
B & 1057 & 813 & 0.769 \\
C & $255 \cdot 4$ & $239 \cdot 3$ & 0.703 \\
D & 889 & 623 & 0.701 \\
\hline
\end{tabular}

performance but it will also increase both latency and memory requirements of the decoder.

\section{Concatenated coding}

A study of the performance of a standard concatenated code in the UWB communication system under consideration is of interest as it is widely available. This channel coding scheme is constructed by using an outer Reed-Solomon (RS) $(255,239)$ code over $G F\left(2^{8}\right)$ and an inner rate-3/4 binary punctured convolutional code (PCC) which is based on the de-facto-standard memory- 6 rate- $1 / 2$ convolutional encoder with generators $(171,133)$.

The outputs of the matched filter are delivered to a conventional ("hard-output") soft-decision Viterbi decoder processes to produce estimated sequences of bits in the inner PCC code. The Viterbi decoding (traceback) length is set to $L=112$ bits. Block interleaving to depth 8 is used between the RS encoder and the PCC encoder.

The outer RS $(255,239)$ code is based on the Galois field $G F\left(2^{8}\right)$ with $p(\alpha)=\alpha^{8}+\alpha^{4}+\alpha^{3}+\alpha^{2}+1$, has minimum Hamming distance equal to 17 , and is capable of correcting, among many other combinations of errors, any single error burst of up to 57 bits. Decoding is accomplished as usual, following deinterleaving of the outputs of the Viterbi decoder and reassembling the 8-bit symbols, by using an errors-only decoder.

\section{Binary-image $(889,623)$ code}

This binary code is constructed from a powerful ReedSolomon $(127,89,39)$ code over $G F\left(2^{7}\right)$ by expressing each element by its 7-bit vector representation in $G(2)^{7}$. The polynomial basis with $p(\alpha)=\alpha^{7}+\alpha^{3}+1$ was used to obtain this vector. This code is capable of correcting numerous combinations of random errors and error bursts, including any combination of up to 19 random errors and any single error burst of up to 127 bits. At the receiver of this scheme, the outputs of the adaptive equalizer are passed through a BPSK demodulator to produce (hard) decisions that are fed to an errors-only decoder.

The parameters of the four coding schemes considered in this paper are summarized in Table I. Although the concatenated RS code scheme $\mathrm{C}$ is much longer than the other schemes, it is included as a reference because of its wide availability and also because of its rate which is practically the same as the TPC coding scheme.

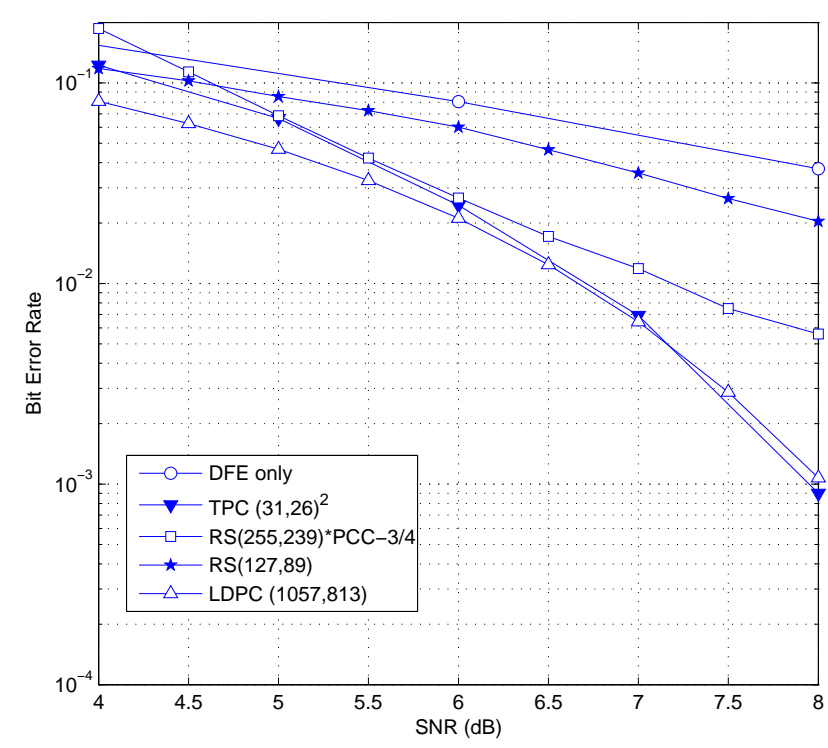

Fig. 9. Error performance of channel coding over a CM3 channel.

\section{Simulation RESUlts}

In this section, simulation results are reported for all the channel coding schemes outlined in the previous section. For each UWB channel type, a total of 10 channel realizations and corresponding BER values over a range of SNR values were generated and the average BER evaluated. Each channel coding scheme is combined with a 95-tap adaptive decisionfeedback equalizer with the parameters given in section III-B.

Figures 9 and 10 show the performances of channel coding over UWB channel types CM3 and CM4, respectively. The case of the CM3 channel illustrates the presence of bad channel realizations that dominate the average. Over both types of channels, the binary-image $(889,623)$ code suffers from a large number of nearest neighbor codewords at minimum Hamming distance and results in worse performance. Both LDPC and TPC codes achieve practically the same performance in the CM3 channel case. On the other hand, over a CM4 channel, best performance is obtained with the regular LDPC $(1057,813)$ code. In this case, both the TPC $(31,26)^{2}$ and the concatenated coding schemes achieve practically the same performance and at a BER value of $10^{-4}$ are at about 1 $\mathrm{dB}$ away from the LDPC code.

\section{CONCLUSIONS}

Adaptive equalization and channel coding schemes applied to pulse-based UWB communication systems in short-range indoor environments have been studied. These offers lowcomplexity alternatives to joint coding and equalization. It has been shown that the performance of an adaptive DFE equalizer is very sensitive to channel conditions in an indoor environment. Simulation results have been presented of coding schemes combined with adaptive equalization to show that this sensitivity is effectively reduced. The TPC $(31,26)^{2}$ code and the regular LDPC $(1057,813)$ code presented in this 


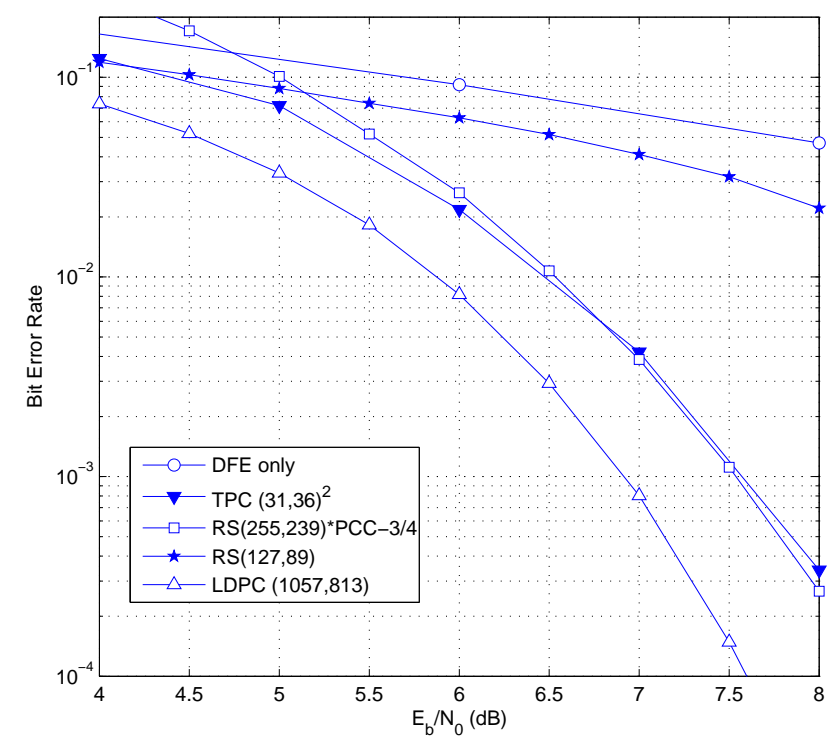

Fig. 10. Error performance of channel coding over a CM4 channel.

paper are good candidates for applications in low-latency lowcomplexity UWB communication systems.

\section{REFERENCES}

[1] H.F. Engler, Jr., "Technical Issues in Ultra-Wideband Radar Systems," Introduction to Ultra-Wideband Radar Systems, 1st ed., Ed. J.D. Taylor. Boca Raton, FL: CRC Press, 1995, Ch. 2.

[2] M.Z. Win and R.A. Scholtz, "Characterization of Ultra-Wide Bandwidth Wireless Indoor Channels: A Communication-Theoretic View," IEEE J. Select. Areas Commun., vol. 20, pp. 1613-1627, Dec. 2002.

[3] C.L. Bennett and G.F. Ross, "Time-domain Electromagnetics and its Applications," Proc. IEEE, vol. 66, pp. 299-318, Mar. 1978.

[4] M.Z. Win and R.A. Scholtz, "Impulse Radio: How it works," IEEE Comm. Lett., vol. 2, pp. 10-12, Jan. 1998.

[5] "Revision of Part 15 of the Commission's Rules Regarding UltraWideband Transmission Systems," FCC, Washington, DC, FCC 02-48, ET Docket 98-153, released Apr. 22, 2002, pp. 26-27.

[6] J. Ellis, K. Siwiak and R. Roberts, "TG3a Technical Requirements," IEEE P802.15-03/030rO, Dec. 27, 2002.

[7] J.R. Foerster, M. Pendergrass and A.F. Molisch, "A Channel Model for Ultrawideband Indoor Communication," TR2003-73, Mitsubishi Electric Research Laboratories, Nov. 2003.

[8] A. Salehand R.A. Valenzuela, "Statistical Model for Indoor Multipath Propagation," IEEE J. Select. Areas Comm., vol. 5, pp. 128-137, Feb. 1987.

[9] R.H. Morelos-Zaragoza, T. Becker and S. Aquino, "A Suboptimal Receiver with Turbo Block Coding for Ultra-Wideband Communications," Proc. IEEE WirelessComm 2005, Maui, Hawaii, June 2005.

[10] J.D. Forney, Jr., "Maximum-Likelihood Sequence Estimation of Digital Sequences in the Presence of Intersymbol Interference," IEEE Trans. Info. Theory, vol. IT-18, no. 3, pp. 363-378, May 1972.

[11] K. Takizawa and R. Kohno, "Low-Complexity Rake Reception and Equalization for MBOK DS-UWB Systems," Proc. IEEE Globecom 2004, Dec. 2004.

[12] S. Qureshi, "Adaptive Equalization," IEEE Comm. Mag., no. 3, vol. 20, pp. 9-16, Mar. 1982.

[13] S. Haykin, Adaptive Filter Theory, 3rd ed. Upper Saddle River, NJ: Prentice Hall, 1996.

[14] P. Monsen, "Adaptive Equalization of the Slow Fading Channel," IEEE Trans. Comm., vol. COM-22, no. 8, pp. 1064-1075, Aug. 1974.

[15] S. Ariyavisitakul and L.J. Greenstein, "Reduced-Complexity Equalization Techniques for Broadband Wireless Channels," IEEE J. Sel. Areas in Comm., vol. 15, no. 1, pp. 5-15, Jan. 1997.
[16] W.H. Gerstacker, R.R. Müller and J.B. Huber, "Iterative Equalization with Adaptive Soft Feedback," IEEE Trans. Comm., vol. 48, no. 9, pp. 1462-1466, Sept. 2000.

[17] E. Baccarelli, A. Fasano and A. Zucchi, "A Reduced-State SoftStatistics-Based MAP/DF Equalizer for Data Transmission over Long ISI Channels," IEEE Trans. Comm., vol. 48, no. 9, pp. 1441-1446, Sept. 2000.

[18] I.J. Febrier, S.B. Gelfand and M.P. Fitz, "Reduced Complexity Decision Feedback Equalization for Multipath Channels with Large Delay Spread," IEEE Trans. Comm., vol. 49, no. 6, pp. 927-937, June 1999.

[19] T.J. Willink, P.H. Wittke and L.L. Campbell, "Evaluation of the Effects of Intersymbol Interference in Decision-Feedback Equalizers," IEEE Trans. Comm., vol. 48, no. 4, pp. 629-636, April 2000.

[20] J.D. Choi and W.E. Stark, "Performance of Ultra-Wideband Communications With Suboptimal Receivers in Multipath Channels," IEEE J. Select. Areas Commun., vol. 20, no. 9, pp. 1754-11766, Dec. 2002.

[21] W.H. Gerstacker and R. Schober, "Equalization Concepts for EDGE," IEEE Trans. Wireless Comm., vol. 1, no. 1, pp. 190-199, Jan. 2002.

[22] S. Lin and D.J. Costello, Jr., Error Control Coding, 2nd ed., PrenticeHall: New Jersey, 2004.

[23] R.M. Pyndhia, "Near-Optimum Decoding of Product Codes: Block Turbo Codes," IEEE Trans. Comm., vol. 46, no. 8, pp. 1003-1010, Aug. 1998.

[24] D. Chase, "A Class of Algorithms for Decoding Block Codes with Channel Measurement Information," IEEE Trans. Info. Theory, vol. IT18, no. 5, pp. 533-547, Sept. 1981.

[25] IEEE Std 802.16-2004, Part 16: Air Interface for Fixed Broadband Wireless Access Systems, IEEE: New York, NY, June 24, 2004.

[26] A.M. Michelson and A.H. Levesque, Error-Control Techniques for Digital Communication, Wiley: New York, 1985.

[27] P.A. Martin and D. P. Taylor, "Distance Based Adaptive Scaling in Suboptimal Iterative Decoding," IEEE Trans. Comm., vol. 50, no.6, June 2002, pp. 869-870.

[28] R.M. Tanner, "A Recursive Approach to Low Complexity Codes," IEEE Trans. Info. Theory, vol. IT-27, no. 5, pp. 170-182, Jan. 1972.

[29] http://www.inference.phy.cam.ac.uk/mackay/CodesFiles.html

[30] J. Pearl, Probabilistic Reasoning in Intelligent Systems, Morgan Kaufman: San Mateo, CA, 1988.

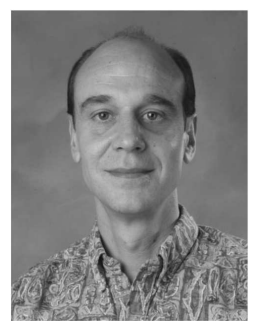

Robert H. Morelos-Zaragoza was born in Houma, Louisiana, U.S.A. He received the BSEE and MSEE degrees from the National Autonomous University of Mexico (UNAM) in 1985 and 1987, respectively, and the $\mathrm{PhD}$ degree in Electrical Engineering from the University of Hawaii at Manoa, in 1992. He was an Assistant Professor at Instituto Tecnológico y de Estudios Superiores de Monterrey (ITESM) of Mexico in 1992-1993. In 1993-1995, he held postdoctoral positions at Osaka University, Japan, and Nara Institute of Science and Technology, Japan.

Dr. Morelos-Zaragoza was a research associate of Imai Laboratory of the University of Tokyo, from 1995 to 1997 . During the period 1997-1999, he was a staff member of the Channel Coding Group of LSI Logic Corporation. In 1999-2002, he was a researcher of the Sony Computer Science Laboratories (Sony CSL) in Tokyo, Japan. Since 2002, he is with the Department of Electrical Engineering of San Jose State University,. His current research interests include error correcting coding, software-defined cognitive radio and wireless communication system design. He is a senior member of IEEE and of Eta Kappa Nu. 